\title{
Urinary metabolic profiling of cisplatin nephrotoxicity and nephroprotective effects of Orthosiphon stamineus leaves elucidated by 1 H NMR spectroscopy
}

\begin{abstract}
Orthosiphon stamineus (OS) is a popular medicinal herb used in traditional Chinese medicine as a diuretic agent and for renal system disorders. This study employed $1 \mathrm{H}$ NMR based metabolomics approach to investigate the possible protective activity of OS in cisplatin induced nephrotoxicity owing to its diuretic and antioxidant activities. Aqueous (OSAE) and $50 \%$ aqueous ethanolic (OSFE) extracts of OS leaves were orally administered at $400 \mathrm{mg} / \mathrm{kg}$ BW doses to rats which were then intraperitoneally injected with cisplatin at $5 \mathrm{mg} / \mathrm{kg} \mathrm{BW}$ dose. The $1 \mathrm{H}$ NMR profile of the urine samples collected on day 5 after cisplatin administration were analyzed by multivariate pattern recognition techniques, whereby 19 marker metabolites suggestive in the involvement of TCA cycle, disturbed energy metabolism, altered gut microflora and BCAA metabolism pathways were identified. It was observed that OSFE caused significant changes $(\mathrm{p}<0.05)$ in the levels of 8 markers namely leucine, acetate, hippurate, lysine, valine, 2-oxoglutarate, 3-HBT and acetoacetate resulting in a moderate ameliorative effect, however, it did not completely protect from nephrotoxicity. OSAE did not demonstrate significant down regulatory effects on any markers, albeit, it potentiated the cisplatin nephrotoxicity by inducing significant increase in glucose, glycine, creatinine, citrate, TMAO, acetate and creatine levels. A Principal Component Analysis (PCA) of the 1H NMR spectra of OS extracts identified that OSFE had higher concentrations of the secondary metabolites such as caffeic acid, chlorogenic acid, protocatechuic acid and orthosiphol, among others. Whereas, OSAE was characterized by higher concentrations of acetate, lactate, succinic acid, valine and phosphatidylcholine. This research denotes the first comprehensive analysis to identify the effects of OS extracts on cisplatin nephrotoxicity.
\end{abstract}

Keyword: Cisplatin nephrotoxicity; Java tea; Metabolomics; OPLS-DA; Orthosiphon stamineus 